\title{
POLARIMETRIC MEASUREMENTS OF THE FINE STRUCTURE OF A SUNSPOT PENUMBRA
}

\author{
J. HOFMANN, F.-L.DEUBNER AND B.FLECK \\ Institut für Astronomie und Astrophysik der Universität Würzburg, Am \\ Hubland, 8700 Würzburg, F.R.Germany \\ W.SCHMIDT \\ Kiepenheuer-Institut für Sonnenphysik, Schöneckstraße 6, 7800 Freiburg, \\ F.R.Germany
}

\begin{abstract}
Observations of a fairly regular sunspot penumbra near disk center have been carried out using a Stokes V polarimeter at the German Vacuum-Tower-Telescope in Izaña, Tenerife. We obtained two-dimensional spectral data by scanning the limb oriented sector of the penumbra in steps of $1^{\prime \prime}$ with the slit parallel to the solar limb. From Stokes V and Stokes I spectra of four magnetic sensitive lines (Fe I 5247.1, Cr I 5247.6, FeI 5250.2, and Fe I 5250.7) obtained with $1024 \times 1024$ pixel CCDs we have determined Doppler shifts and several magnetic parameters. The results of a correlation analysis point out a clear relation between continuum brightness and magnetic field inclination in the sense of steeper field lines in the bright penumbral filaments. There is also a doubtless connection between intensity and Doppler shift. We did find indications for a correlation between field strength and brightness which is not uniform across the penumbra, though.
\end{abstract}

\section{INTRODUCTION}

The magnetic field configuration of sunspot penumbrae has been investigated in many analyses. E.g. Beckers and Schröter (1969) already gave a rather detailed description. Many of their findings are still valid today. Among other things, they supposed the inclination in the dark penumbral filaments to be more horizontal. They also found a downflow in the darker structures. In a recent paper, Degenhardt and Wiehr (1991) presented observational evidence for the proposed relation between continuum brightness and magnetic field inclination, which has been confirmed by Schmidt et al. (1992).

The connection between the field strength and the filamentary structure seems to be somewhat more intricate. Several authors (e.g. Wiehr and Stellmacher 1989; Lites et al. 1990) claimed that there is actually no correlation at spatial scales down to the resolution limit of their observations (typically $0.4^{\prime \prime}$ $\left.0.8^{\prime \prime}\right)$. This is in contrast to Beckers and Schröter (1969) who found an enhanced 
field strength in the darker filaments.

Most of the previous investigations are based on selected high quality spectra that cannot provide conclusive evidence in a statistical sense, however. Of course, the lack of real good seeing over a longer period limits the number of excellent spectra available. We present a statistical analysis based on 48 spectra with good spatial resolution, and we use correlation techniques to examine the above relations.

\section{OBSERVATIONS}

The observations have been carried out at the German VTT in Izaña, Tenerife, in November 1990. The observed sunspot was located near disk center $\left(\theta=10^{\circ}, \mu=0.985\right)$. We used two $1024 \times 1024$ pixel CCD cameras to record simultanously two seperate wavelength ranges (Fe $I 5250.216 \AA$, Fe $I 5250.654 \AA$ and $\mathrm{Fe} I 5247.058 \AA$, $\mathrm{Cr} I 5247.574 \AA$ ). Using a Stokes V polarimeter consisting of a $\lambda / 4$-plate and two crossed calcites we obtained strictly simultanous (I+V) and (I-V) spectra. The observed area covering $40^{\prime \prime} \times 10^{\prime \prime}$ of the penumbra was scanned with the slit oriented parallel to the solar limb and almost perpendicular to the penumbral filaments. The exposure time was $1 \mathrm{sec}$ and the intervall between two subsequent frames was $12 \mathrm{sec}$. We scanned the penumbra 28 times, each scan consisting of 10 frames with a spatial distance of $1^{\prime \prime}$ between following frames.

\section{CORRELATION ANALYSIS}

For our correlation analysis we used the best 48 spectra out of 280 from 28 raster scans of the penumbra. The positions of selected spectra have been chosen to maintain a fairly uniform coverage of the scanned area. To emphasize the interesting small scale fluctuations and to suppress large scale variations all spectra have been subjected to a high pass Fourier filter with a cut off frequency of $3^{\prime \prime}$. Then we determined the crosscorrelation coefficient of the corresponding parameters.

Fig. Ia shows the result of the correlation analysis of continuum brightness and magnetic field strength. The correlation coefficients are ordered by the position number within a particular scan, where 1 corresponds to the slit position at the inner penumbral border and 10 marks the outermost slit position near the spot boundary. We find a gradient in the radial direction indicating a (positive) correlation between brightness and field strength for the inner part of the penumbra and a significant anticorrelation for the outer part. This is in contrast to Wiehr and Stellmacher (1989) and Lites et al. (1990), who did not find any relation between these quantities. We have to point out that we are not sure to fully resolve single filaments, so on smaller scales we may find different results, which cannot be detected within this study.

The relation between inclination and continuum intensity displayed in Fig. Ib shows a clear positive correlation. Here we correlated the ratio (Stokes V)/B, which is proportional to $\cos \gamma$, and continuum brightness. A positive coefficient implies that the angle $\gamma$ between the magnetic field vector and the line of sight is 

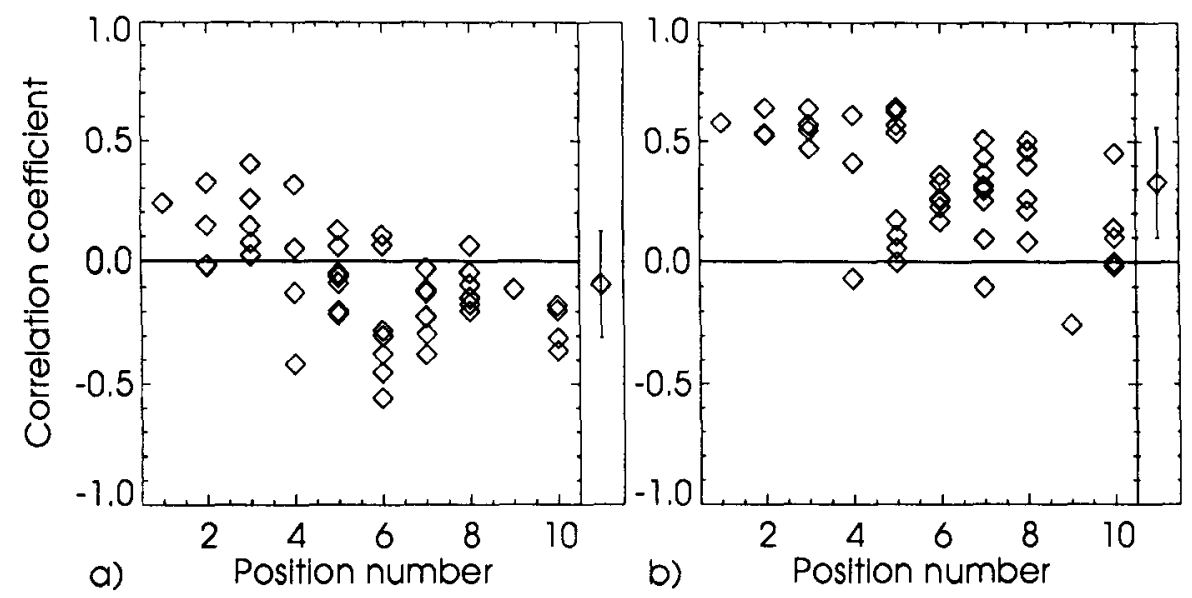

FIGURE I Each correlated data set (frame) is represented by a mark, indicating the particular coefficient of the crosscorrelation. The left panel shows the coefficients for the brightness-field strength correlation determined with $\mathrm{Fe} 5250.2$, the right panel the brightnessinclination correlation (Fe 5250.7). At the right of each panel we have plotted the corresponding mean value with its standard deviation indicating the scatter of the measured coefficients.

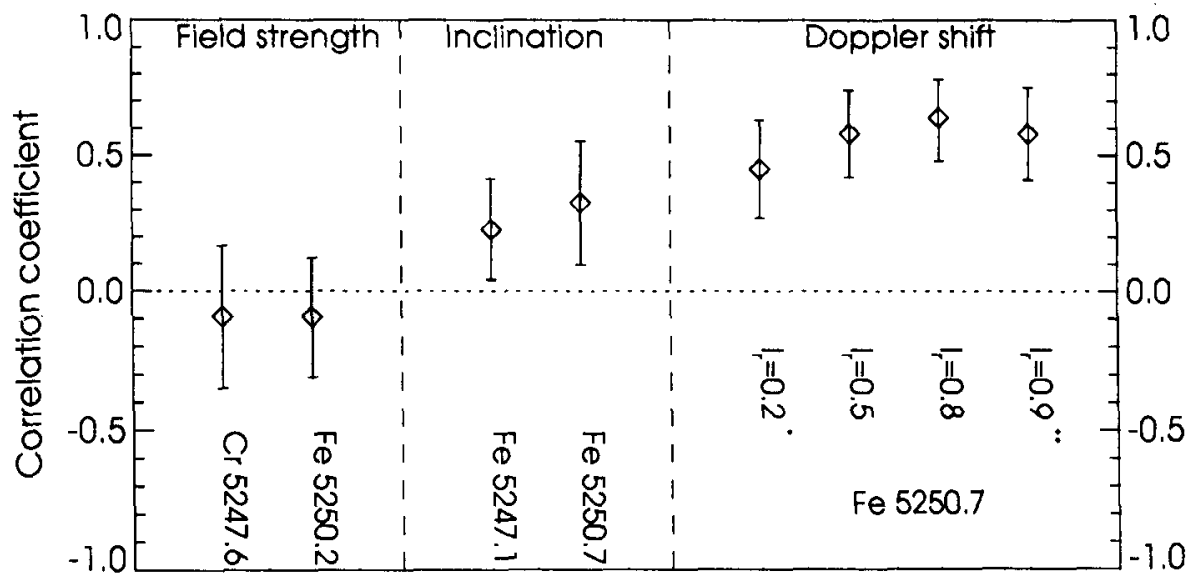

FIGURE II Mean values of the correlation analyses of several quantaties (indicated at the top) with continuum intensity measured for different spectral lines are displayed. The Doppler shifts (blue shift $=$ positive) at the right have been determined at four different relative Intensities $I_{r}=\left(I-I_{0}\right) /\left(I_{\text {cont }}-I_{0}\right)$ according to different hights in the atmosphere (e.g. * line core $\hat{=}$ high layer, ${ }^{* *}$ far wing $\hat{=}$ near $\tau_{\text {cont }}=1$ ). 
smaller at positions with higher continuum intensity. This confirms the results of Degenhardt and Wiehr (1991) and Schmidt et al. (1992).

Fig. II gives an overview of the correlations of field strength and inclination angle with continuum brightness calculated for several spectral lines. Besides, in Fig. II the correlation of Doppler shift and continuum brightness is shown at four different relative intensities $I_{r}=\left(I-I_{0}\right) /\left(I_{\text {cont }}-I_{0}\right)$ corresponding to four different heights in the atmosphere. As result we obtain a very good correlation between Doppler shifts and brightness in the sense that we find red shifts in the dark regions and blue shifts for bright regions (see also Schröter et al. 1989). Quite similar results are found with the other observed lines.

With an inclination angle of $60^{\circ}$ to $80^{\circ}$ with respect to the line of sight, as determined for the observed part of the penumbra, the Evershed outflow which is accepted to be located in the dark streaks (e.g. Beckers 1981; Küveler and Wiehr 1985) should lead to an enhanced blue shift in the dark regions. We can interpret our opposite finding as a superposition of the outflow and a more pronounced downflow in the dark filaments as proposed for the penumbral model of floating filaments (Schmidt et al. 1986).

The large scatter of the correlation coefficients observed demonstrates the advantage of the utilization of a larger sample as compared with single spectrum analysis. Moreover it is important to consider radial variations of the correlations as seen in Fig. Ia. In particular, a reversal of the correlation as the one between field strength and brightness may cause both positive and negative correlations between these two parameters in a single frame (c.f. Fig. Ia), which may lead to wrong conclusions.

\section{REFERENCES}

Beckers, J.M., and Schröter, E.H. 1969, Solar Phys., 10, 384

Beckers, J.M. 1981, in The Sun as a Star, S. Jordan, NASA, Washington, 11

Degenhardt, D., and Wiehr, E. 1991, A\&A, 252, 821

Küveler, G., and Wiehr, E. 1985, A\&A, 142, 205

Lites, B.W., Scharmer, G.B., and Skumanich, A. 1990, A\&A, 355, 329

Schmidt, H.U., Spruit, H.C., and Weiss, N.O. 1986, A\&A, 158, 351

Schmidt, W., Hofmann, A., Balthasar, H., Tarbell, T.D., and Frank, Z.A. 1992, A\&A, 264, L27

Schröter, E.H., Kentischer, T., and Münzer, H. 1989, in High Spatial Resolution Solar Observations, O. v.d. Lühe, Sacramento Peak Observatory, 299

Wiehr, E., and Steilmacher, D. 1989, A\&A, 225, 528 